\title{
Minimizing the infected peak utilizing a single lockdown: a technical result regarding equal peaks
}

\author{
James M. Greene ${ }^{1,2}$ Eduardo D. Sontag 2,3
}

June 26, 2021

\begin{abstract}
Due to the usage of social distancing as a means to control the spread of the novel coronavirus disease COVID-19, there has been a large amount of research into the dynamics of epidemiological models with time-varying transmission rates. Such studies attempt to capture population responses to differing levels of social distancing, and are used for designing policies which both inhibit disease spread but also allow for limited economic activity. One common criterion utilized for the recent pandemic is the peak of the infected population, a measure of the strain placed upon the health care system; protocols which reduce this peak are commonly said to "flatten the curve." In this work, we consider a very specialized distancing mandate, which consists of one period of fixed length of distancing, and addresses the question of optimal initiation time. We prove rigorously that this time is characterized by an equal peaks phenomenon: the optimal protocol will experience a rebound in the infected peak after distancing is relaxed, which is equal in size to the peak when distancing is commenced. In the case of a non-perfect lockdown (i.e. disease transmission is not completely suppressed), explicit formulas for the initiation time cannot be computed, but implicit relations are provided which can be pre-computed given the current state of the epidemic. Expected extensions to more general distancing policies are also hypothesized, which suggest designs for the optimal timing of non-overlapping lockdowns.
\end{abstract}

\section{Introduction}

The ongoing global COVID-19 (coronavirus disease 2019) pandemic, caused by SARS-CoV-2 (severe acute respiratory coronavirus 2), has necessitated the use of non-pharmaceutical interventions (NPIs) as a means to slow transmission of the disease. Although controversial, there is clear evidence that NPIs such as social distancing have saved millions of lives globally [1]. Social distancing mandates, denoted in this manuscript as "lockdowns," cannot be implemented indefinitely, as it carries both a high economic [2,3] and psychological [4] cost. Furthermore, a lack of compliance may make extended protocols unfeasible to implement $[5,6]$. Hence there is a need to optimize the timing of prescribed lockdowns. The optimization of such schedules is the focus of this work. Specifically, we characterize the implementation time of a single non-strict lockdown, with fixed transmission reduction, which minimizes the peak of the infected population in the Susceptible-Recovered-Removed (SIR) model. The main contribution is Theorem 1, which we term an equal peak phenomenon.

There have been a large number of mathematical analyses applied to the spread of COVID-19. In this work, as we solve a small technical problem, we do not attempt to provide a comprehensive literature review. We do however note a number of closely related works based on optimal social distancing strategies. The analysis presented here is a direct extension of [7], where the case of multiple fixed-length non-overlapping complete (i.e. zero disease transmission) lockdowns is completely characterized as a linear programming problem; this manuscript should be viewed as a direct extension of this previous work. Again minimizing the infected peak, the authors of [8] determine the optimal (again, possibly complete) lockdown schedule as a feedback mechanism. Numerical results for a variety of epidemic objectives with respect to a

\footnotetext{
${ }^{1}$ Department of Mathematics, Clarkson University, Potsdam, NY, United States

${ }^{2}$ Department of Electrical and Computer Engineering and Department of Bioengineering, Northeastern University, Boston, MA, United States

${ }^{3}$ Laboratory of Systems Pharmacology, Program in Therapeutic Science, Harvard Medical School, Boston, MA, United States
} 
single interval of distancing are provided in $[9,10]$, and [11] studies the same problem both numerically and theoretically. A constrained optimization problem is solved in [12], where the time minimal distancing policy which maintains an upper bounded on the infected population is derived. There are also a number of works which minimize the total number of infections during an epidemic during a period of such distancing; this is studied numerically in [9] and analytically in $[13,14,15]$. Interestingly, the main result of [13] is that the optimal lockdown policy to minimize the total number of infected individuals coincides with the protocol considered in this work; see equation (4) below. However, as observed in [9], the timing with respect to these differing objectives (minimizing infected peak vs. minimizing total number of infections) in general do not agree, so that policy makers cannot generally hope to achieve both simultaneously.

This work is organized as follows. In Section 2, we recall the SIR model and precisely formulate the social distancing protocol to be optimized. Results are presented in Section 3, and a discussion with potential (unproven) extensions are postulated in Section 4. Some preliminary numerical simulations are also provided in Section 4. Proofs of all results are provided in Section 5.

\section{Problem formulation}

We consider the classic SIR epidemic model introduced by Kermack and McKendrick in 1927 [16], which we briefly review here. The ordinary differential equations (ODEs) describing the evolution of the system are given below:

$$
\begin{aligned}
\frac{d S}{d t} & =-\beta(t) S I \\
\frac{d I}{d t} & =\beta(t) S I-\nu I \\
\frac{d R}{d t} & =\nu I,
\end{aligned}
$$

together with initial conditions

$$
\begin{aligned}
S(0) & =S_{0} \\
I(0) & =I_{0} \\
R(0) & =0 .
\end{aligned}
$$

Here $S$ denotes the susceptible population, $I$ the infected population, and $R$ the removed population, where the latter combines those individuals that have either obtained immunity or died. Parameter $\nu$ represents the combined recovery and death rate of the disease, and hence if mortality is relatively small, is a measure of the rate of recovery to immunity. We assume that $\nu$ is constant. Parameter $\beta=\beta(t)$ quantifies the transmission rate between susceptible and infected individuals. We consider this a time-varying parameter, since NPIs are generally viewed as altering this transmission rate. Specifically, during a lockdown, where contacts are reduced and/or mask mandates are enforced, the transmission rate may be modeled as decreasing by a factor of $p$, where $0 \leq p<1$ :

$$
\beta \stackrel{\text { lockdown }}{\longrightarrow} p \beta .
$$

For example, in [17], estimated reductions in $\mathcal{R}_{0}$ (which for the SIR model is equivalent to reducing $\beta$; see equation (7) below) yield $p$ values as large as 0.58 , with this value corresponding to mandates limiting gatherings to 10 people or less.

We are specifically interested in the effect of a single fixed period of social distancing (i.e. a single lockdown) on the peak of the infected population:

$$
I_{\max }:=\max _{t \in[0, \infty)} I(t) .
$$

Our goal is thus to understand the behavior of $I_{\max }$ as a function of $\beta(t)$, where $\beta(t)$ take the following form:

$$
\beta(t)= \begin{cases}\beta, & 0 \leq t \leq t_{s} \\ p \beta, & t_{s}<t \leq t_{s}+T \\ \beta, & t_{s}+T \leq t\end{cases}
$$




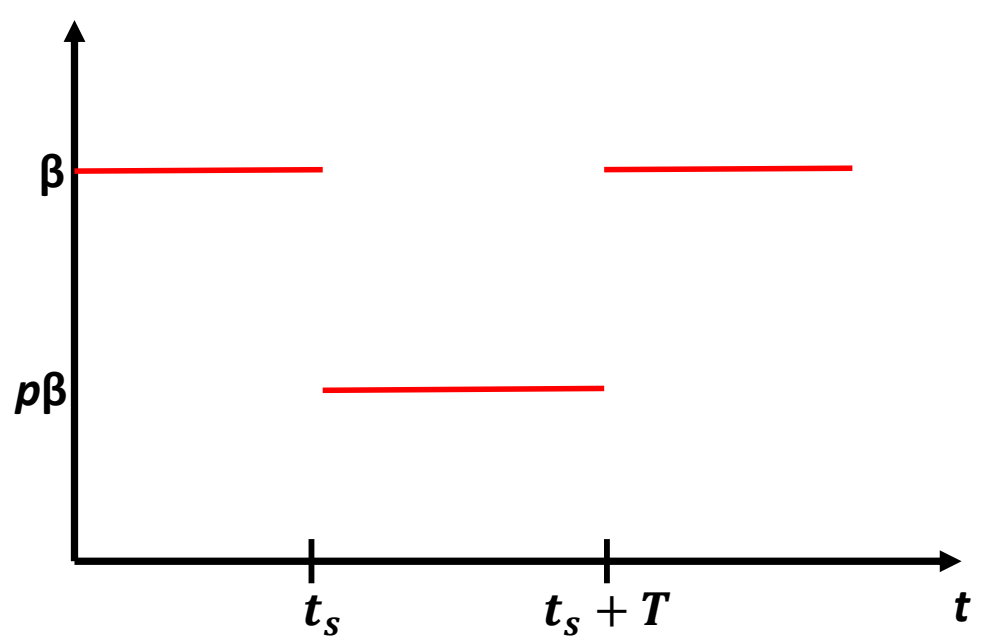

Figure 1: Visualization of idealized lockdown (4). We assume that transmission is reduced by an effective amount $p$ during the lockdown, where $0<p<1$. The lockdown is initiated at $t_{s}$ and is enacted for $T$ units of time.

As discussed above, $0 \leq p<1$ represents the reduction in transmission rate due to distancing mandates, which are enacted at time $t_{s}$ for a length of time $T$. That is, a lockdown occurs for $t \in\left[t_{s}, t_{s}+T\right]$. For a visualization of the lockdown protocol, see Figure 1. We assume that $\beta, \nu, p$, and $T$ are fixed and known, and we are interested in optimizing the start time $t_{s}$ of distancing so as to minimize the infected peak as a function of $t_{s}$ :

$$
\min _{t_{s} \in[0, \infty)}\left\{I_{\max }\left(t_{s}\right)\right\} .
$$

It is intuitively clear that $t_{*}$ should not occur too early or too late: begin too early and we simply delay the full effect of the epidemic, and begin too late and the epidemic has already passed throughout the susceptible population, and hence social distancing has minimal impact. It is the goal of this work to understand the optimal timing with respect to the metric (5), with transmission rate of the form (4). This problem is a generalization of [7], which studied the same problem for $p=0$. In general we allow $p>0$ in the following.

We lastly note the maximum appearing in (3) is indeed a maximum (i.e. it is achieved at some time, as opposed to a supremum), since $I$ is continuous and $I(t) \rightarrow 0$ as $t \rightarrow \infty$ for all distancing protocols $\beta(t)$. For more details and a proof, see for example [18].

We assume that the populations are normalized, so that

$$
S_{0}+I_{0}=1 .
$$

Assumption (6) ensures that $S(t)+I(t)+R(t)=1$ for all $t \geq 0$, i.e. that variables $S, I$, and $R$ represent population fractions. System (1) is also seen to be positively invariant. Note lastly that the removed population $R$ does not affect the dynamics of the above system, but may serve as a useful measure of disease progression.

\subsection{Additional assumptions}

In this section, we impose additional assumptions on the model introduced in Section 2. We note that these assumptions are not crucial for the following theory, but instead are useful for limiting the number of potential cases which we feel would otherwise obfuscate the exposition. Specifically, they allow us to conclude with certainty the exact locations of the potential relative maxima of the infected population curves, while excluding particular "boundary cases".

Our first additional assumption is that the lockdown has the ability to immediately stop disease progression independently of the start time. In other words, we assume that the transmission rate is able to be reduced such that for any start time $t_{s}$,

$$
\frac{d I}{d t}(t) \leq 0 \text {. }
$$


for all $t \in\left[t_{s}, t_{s}+T\right]$. Since on this time interval

$$
\frac{d I}{d t}=\nu\left(\frac{p \beta}{\nu} S-1\right) I,
$$

this assumption is equivalent to

$$
\frac{p \beta}{\nu} S_{0}<1,
$$

as $S$ is non-increasing on $[0, \infty)$. Defining the basic reproduction number $\mathcal{R}_{0}$ as

$$
\mathcal{R}_{0}(\beta):=\frac{\beta}{\nu},
$$

the previous assumption is equivalent to

$$
p<\frac{1}{\mathcal{R}_{0}(\beta) S_{0}},
$$

or again equivalently

$$
\mathcal{R}_{0}(p \beta) S_{0}<1 .
$$

Furthermore, for an epidemic to exist (i.e. for $I$ to increase at any time point, which is equivalent to increasing initially, since $S$ is non-increasing), we must assume that $\dot{I}(0)>0$. Examining the second of (1) (together with $\beta(t)=\beta$ and the definition of $\mathcal{R}_{0}(\beta)$ ), this implies that

$$
\mathcal{R}_{0}(\beta) S_{0}>1
$$

Thus, for the remainder of the manuscript, we assume the following:

$$
\begin{gathered}
\mathcal{R}_{0}(p \beta) S_{0}<1 \\
\mathcal{R}_{0}(\beta) S_{0}>1 .
\end{gathered}
$$

\section{Results}

\subsection{Infected peak formulas}

The assumptions presented in Section 2.1 imply that the graph of $I:[0, \infty) \rightarrow[0,1]$ may have at most two local maxima, one of which must be global. In fact, there are exactly two possible cases, dependent on the lockdown initiation time $t_{s}$ :

1. $I$ has a unique local maximum $I_{\max }$ occurring on $\left[0, t_{s}\right]$.

2. $I$ has one maxima at $t_{s}\left(I\left(t_{s}\right)\right)$, and another local maximum on $\left[t_{s}+T, \infty\right)$.

Note that Case 2 occurs precisely because of the second assumption in (8), i.e. since $I$ is non-increasing on $\left[t_{s}, t_{s}+T\right]$. More precisely, Case 2 occurs for $t_{s}$ such that

$$
\mathcal{R}_{0}(\beta) S\left(t_{s}+T\right)>1,
$$

which in words means that $I$ initially increases after the lockdown is released (i.e. at time $t_{s}+T$ ). Since $S$ is nonincreasing, (9) implies that

$$
\mathcal{R}_{0}(\beta) S\left(t_{s}\right)>1
$$

i.e. that $I$ was also increasing prior to lockdown initiation.

It is not hard to see that the maximum in Case 1 is always larger than the pair of maxima in Case 2, so that the minimization problem is solved by $t_{s}$ of the form of Case 2 . In fact, we have the following proposition. 
Proposition 1. Assume that the lockdown initiation time $t_{s}$ is such that two relative maxima of I exist. Then the second maxima, occurring at some time $t \in\left[t_{s}+T, \infty\right)$, is given by

$$
I_{p}\left(t_{s}\right):=V_{0}-\frac{1-p}{p} \cdot \frac{1}{\mathcal{R}_{0}(\beta)} \log \left(\frac{S\left(t_{s}\right)}{S\left(t_{s}+T\right)}\right)
$$

where

$$
V_{0}:=I_{0}+S_{0}-\frac{1}{\mathcal{R}_{0}(\beta)}\left(1+\log \left(\mathcal{R}_{0}(\beta) S_{0}\right)\right) .
$$

Furthermore, $V_{0}$ is the maximum of I corresponding to the case of a unique global maximum occurring in $\left[0, t_{s}\right]($ Case 1 above). Thus, the minimization problem (5) is solved for $t_{s}$ such that I admits two relative maxima (Case 2 above).

Recall that we are assuming that $0<p<1$, which implies $S\left(t_{s}+T\right)<S\left(t_{s}\right)$. Since $I\left(t_{s}\right) \leq V_{0}$ and the second term on the right-hand side of (11) is positive, we have that (5) is solved for $t_{s}$ corresponding to Case 2 above, as claimed.

Before undertaking an analysis of $I_{p}\left(t_{s}\right)$, we note that there are several equivalent representations of the second peak $I_{p}$. By the change of variables $S=S(t)$, we see that

$$
\begin{aligned}
\log \left(\frac{S\left(t_{s}+T\right)}{S\left(t_{s}\right)}\right) & =\int_{S\left(t_{s}\right)}^{S\left(t_{s}+T\right)} \frac{\mathrm{d} S}{S} \\
& =\int_{t_{s}}^{t_{s}+T} \frac{1}{S(t)} \frac{d S}{d t}(t) \mathrm{d} t \\
& =\int_{t_{s}}^{t_{s}+T} \frac{1}{S(t)}(-p \beta S(t) I(t)) \mathrm{d} t \\
& =-p \beta \int_{t_{s}}^{t_{s}+T} I(t) \mathrm{d} t,
\end{aligned}
$$

since $\dot{S}=-p \beta S I$ on $\left(t_{s}, t_{s}+T\right)$. Hence we can write (11) in the following form:

$$
\begin{aligned}
I_{p}\left(t_{s}\right) & =V_{0}-(1-p) \beta \cdot \frac{1}{\mathcal{R}_{0}(\beta)} \int_{t_{s}}^{t_{s}+T} I(t) \mathrm{d} t+ \\
& =V_{0}-(1-p) \nu \int_{t_{s}}^{t_{s}+T} I(t) \mathrm{d} t
\end{aligned}
$$

Since $\dot{R}(t)=\nu I(t)$, we can also write the previous integral as

$$
\begin{aligned}
\int_{t_{s}}^{t_{s}+T} I(t) \mathrm{d} t & =\frac{1}{\nu} \int_{t_{s}}^{t_{s}+T} \dot{R}(t) \mathrm{d} t \\
& =\frac{1}{\nu}\left(R\left(t_{s}+T\right)-R\left(t_{s}\right)\right) .
\end{aligned}
$$

Thus, another equivalent form for $I_{p}\left(t_{s}\right)$ is given by

$$
I_{p}\left(t_{s}\right)=V_{0}-(1-p)\left(R\left(t_{s}+T\right)-R\left(t_{s}\right)\right) .
$$

\subsection{Analysis of relative maxima of $I$}

Under assumptions (8), Proposition 1 implies that to minimize the peak of the infected population with respect to a lockdown represented by (4), we must minimize both relative maxima $I\left(t_{s}\right)$ and $I_{p}\left(t_{s}\right)$ simultaneously with respect to $t_{s}$. Recall that $I\left(t_{s}\right)$ denotes the infected population at the onset of the lockdown (i.e. $I$ at time $\left.t_{s}\right)$, and $I_{p}\left(t_{s}\right)$ is the relative maxima of $I$ occurring at some $t \in\left[t_{s}+T, \infty\right)$, i.e. after the lockdown has been lifted. Specifically we note that we do 
not have an explicit formula for the time $I_{p}$ occurs, and the notation is meant to emphasize that $I_{p}$ depends on $t_{s}$ via the expression (11).

Numerical simulations for a specific set of $\beta, p, \nu, S_{0}$ and $I_{0}$ are provided in Figure 2. Here we simply vary the start time $t_{s}$ for the distancing protocol represented by (4), and plot representative infection response dynamics in Figure 2a. Note that if the distancing starts too early (e.g. $t_{s}=20$ days), then the peak of the infected population $I_{\max }$ is simply delayed until after the lockdown is lifted; $V_{0} \approx 0.4037$ for the set of parameters in Figure 2. Similarly, if the lockdown is initiated too late (e.g. $t_{s}=70$ days), then the distancing mandate has only a marginal effect on reducing $I_{\max }$. For intermediate $t_{s}$, we observe two relative maxima $I\left(t_{s}\right)$ and $I_{p}\left(t_{s}\right)$, as discussed in the beginning of this section. It appears that as $t_{s}$ is increased, the first peak $I\left(t_{s}\right)$ increases, while the second peak $I_{p}\left(t_{s}\right)$ decreases. This is intuitive, since increasing $t_{s}$ allows us to initiate the lockdown closer to $V_{0}$ (increasing $I\left(t_{s}\right)$ ), which at the same time builds immunity in the population and hence decreases the magnitude of the "second wave" (which is quantified by $I_{p}\left(t_{s}\right)$ ). Furthermore, it appears that the optimal choice of $t_{s}$ balances these two effects precisely:

the minimizer $t_{s}$ of $I_{\max }$ is the unique start time such that $I\left(t_{s}\right)=I_{p}\left(t_{s}\right)$, i.e. the two relative maxima of I are equal

This hypothesis is generally true, and is stated precisely in the following theorem.

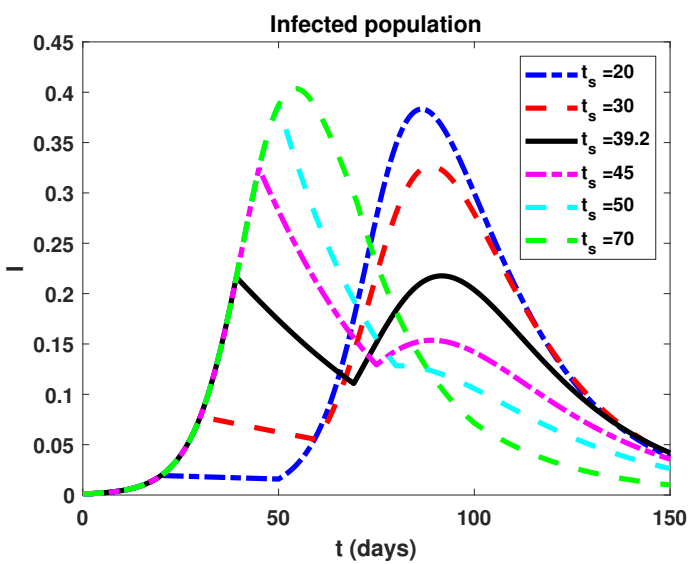

(a) Epidemic response to various $t_{s}$

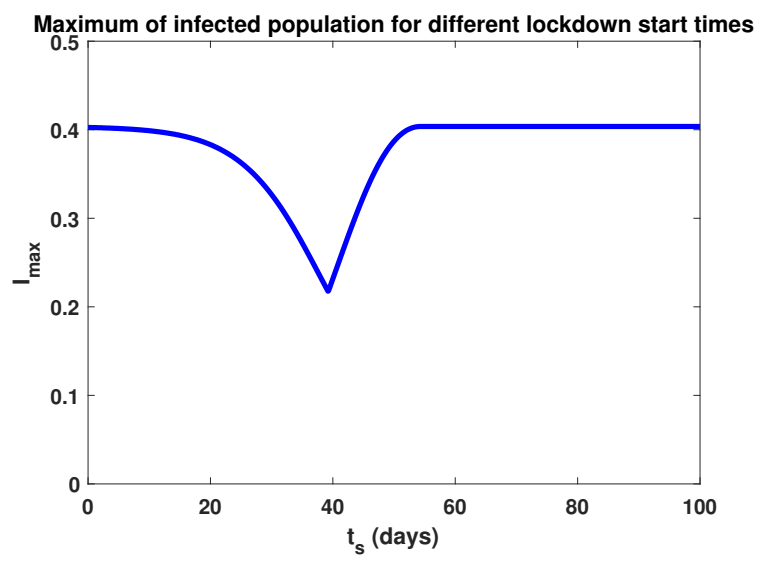

(b) $I_{\max }$ as a function of $t_{s}$

Figure 2: Response of infected population to a single lockdown as characterized by (4). The following parameters were utilized in the above simulation: $\beta=0.2, p=0.2252, \nu=0.05, I_{0}=10^{-4}$, and $S_{0}=1-I_{0}$. Note that according to (7), $\mathcal{R}_{0}=4$ prior to lockdown, while $\mathcal{R}_{0} \approx 0.9$ during the lockdown. We observe a global minimum of $I_{\max }$ at $t_{s} \approx 39.2$ days, which is corresponds to the black curve in Figure 2a. Note that both relative maxima appear to be equal for this minimizing $t_{s}$.

THEOREM 1. Consider the SIR epidemic model (1) with time-varying transmission rate (4) representing a single lockdown of relative efficacy $p$ and fixed duration $T$. Under assumptions (8), the optimal time $t_{s}$ of lockdown initiation to minimize the peak of the infected population is such that I has two relative maxima of equal size. In this case, the two maxima of $I$ are precisely $I\left(t_{s}\right)$ and $I_{p}\left(t_{s}\right)$.

A proof of Theorem 1 is postponed until Section 5. Theorem 1 thus says that the optimal choice of $t_{s}$ to "flatten the curve" is such that the infected population will have precisely two relative maxima of equal size: one of which occurs at lockdown initiation $\left(t_{s}\right)$, and the other given by a second wave occurring after the lockdown has been released (after $\left.t_{s}+T\right)$. We call this an equal peaks phenomena, with an unavoidable second wave of infections which will rebound to the same maximum intensity as experienced prior to the lockdown. We note that such a phenomenon occurs only for optimally designed interventions (indeed, it is a characterization), and it is possible to choose $t_{s}$ large enough to reduce the second peak, or even remove it entirely (see the $t_{s}=70$ days curve in Figure 2a). However, such distancing mandates will necessarily lead to a larger first peak in infections. Similarly, the first peak can be made as small as $I_{0}$ if $t_{s}$ is initiated early, but in this case a large second wave is encountered (see the $t_{s}=20$ days curve in Figure 2a). 
The result of Theorem 1 provides an implicit method of determining the optimal lockdown initiation time given the current state of the epidemic. The initiation time $t_{s}$ is characterized by the relation

$$
I\left(t_{s}\right)=I_{p}\left(t_{s}\right) .
$$

Utilizing (14) together with the (normalized conservation law)

$$
R(t)=1-S(t)-I(t),
$$

equation (15) can be written as

$$
\left.I\left(t_{s}\right)+(1-p)\left(S\left(t_{s}\right)-S\left(t_{s}+T\right)+I\left(t_{s}\right)-I\left(t_{s}+T\right)\right)\right)=V_{0},
$$

which yields an implicit relation to determine $t_{s}$. Note that the time $t_{s}$ cannot be computed explicitly (unlike the formulas which appear in [7]), since when $p>0$ there are no analytic solutions to the SIR system in $\left[t_{s}, t_{s}+T\right]$. But of course, as the epidemic evolves, the relation (16) can be tested numerically. More precisely, policy could be designed by utilizing current epidemic data, assuming $t_{s}=0$, and checking whether relation (16) is currently satisfied (with uncertainty sufficiently quantified, and assuming good estimates for lockdown efficacy $p$ exist). If this equation is satisfied, the lockdown should be initiated as soon as possible. We finally note that other relations similar to (16) exist, which utilize the alternate forms of $I_{p}$ presented in Section 3.1, as well as the conserved quantity $H(S, I)$ to compute $I\left(t_{s}\right)$ as a function of $S\left(t_{s}\right)$ and the initial data (see the proof of Proposition 1 in Section 5). Specifically, a relation only involving $S\left(t_{s}\right)$ and $S\left(t_{s}+T\right)$ is given as follows:

$$
1+\log \left(\mathcal{R}_{0}(\beta)\right)+\log \left(S\left(t_{s}\right)\right)-\mathcal{R}_{0}(\beta) S\left(t_{s}\right)+\frac{1-p}{p} \log \left(\frac{S\left(t_{s}\right)}{S\left(t_{s}+T\right)}\right)=0 .
$$

\section{Discussion and extensions}

In this work we have proven a characterization of the optimal start time with respect to (5) for a lockdown of fixed length $T$ and transmission reduction factor $p$ in the SIR model. This characterization is classified according to an equal peaks phenomenon: the infection response will exhibit two local maxima of equal magnitude. The result (Theorem 1) was proven under certain assumptions (8), but these were assumed for clarity of exposition, and the result remains valid under weaker hypothesis. In particular, if the lockdown is not "strong enough" (i.e. $p$ does not satisfy the first of (8)), then the first relative maxima of $I$ may occur interior to the lockdown interval $\left[t_{s}, t_{s}+T\right]$, and not at $t_{s}$. But the result of Theorem 1 remains true: the optimal initiation time is such that both relative maxima are equal, i.e. the response of $I$ possesses equal peaks.

The results of this work are concerned with optimizing the initiation time of a single non-perfect lockdown. In reality, social distancing directives are not designed utilizing a single interval of distancing, but more generally consist of multiple periods of possibly different levels of mandated distancing, which in the above model, correspond to different transmission reduction factors $p$. We conjecture that the above equal peaks phenomenon generalizes to the case of multiple lockdowns. More precisely, for $k=1,2, \ldots, n$ define disjoint intervals

$$
J_{k}:=\left[t_{s_{k}}, t_{s_{k}}+T_{k}\right]
$$

and $\beta(t)$ be the time-varying transmission rate

$$
\beta(t)= \begin{cases}p_{k} \beta, & t \in J_{k} \\ \beta, & t \notin \cup_{k=1}^{n} J_{k} .\end{cases}
$$

Here $\beta(t)$ represents a series of $n$ lockdowns, each of fixed (but generally different) length $T_{k}$ and transmission reduction factor $p_{k}$. A visualization for $n=2$ is provided in Figure 3. We then can consider an optimization problem analogous to (5), where we minimize the peak of the infected population with respect to the start times $t_{s_{k}}$ of the $k=1,2, \ldots, n$ lockdowns (assuming all other parameters are fixed and known). The conjecture generalizing Theorem 1 is then that the initiation times are such that the infected population exhibits $n+1$ relative maxima, each of equal size; that is, it possesses 


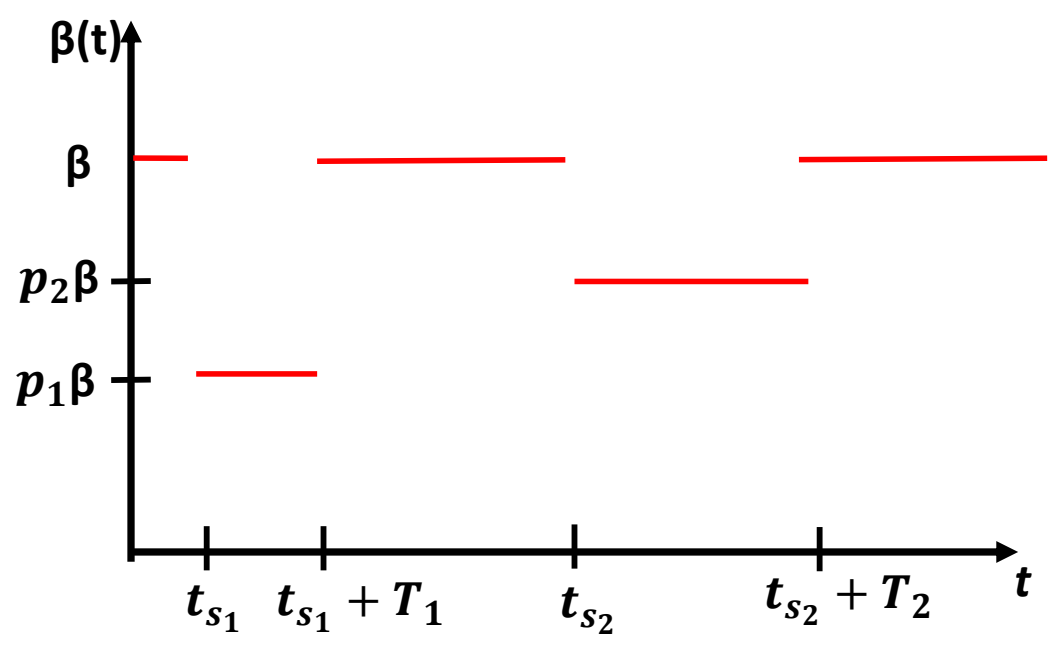

Figure 3: Visualization of $n=2$ lockdowns (17). We assume that transmission is reduced by an effective amount $p_{1}$ during $\left[t_{s_{1}}, t_{s_{1}}+T_{1}\right]$ and $p_{2}$ during $\left[t_{s_{2}}, t_{s_{2}}+T_{2}\right]$, where $0<p_{1}, p_{2}<1$.

$n+1$ equal peaks. Although we do not prove the result here, numerical simulations seem to suggest its validity. A numerical experiment for $n=2$ is provided in Figure 4a. Here we simply iterate over all possible intervals $\left[t_{s_{1}}, t_{s_{1}}+T_{1}\right]$ and $\left[t_{s_{2}}, t_{s_{2}}+T_{2}\right]$ that do not overlap, as we cannot start a second lockdown before the first one has ended; these prohibited times correspond to the white region in Figure $4 \mathrm{a}$, and is given parametrically by $t_{s_{2}} \geq 0, t_{s_{1}}-T_{2}<t_{s_{2}}<t_{s_{1}}+T_{1}$ in the first quadrant. Bluer shades in Figure 4a correspond to smaller infection peaks, and the approximate optimal policy together with the infection response is provided in Figure $4 \mathrm{~b}$. Note that the infected population exhibits $3=n+1$ relatives maxima (peaks) of equal size, as hypothesized.

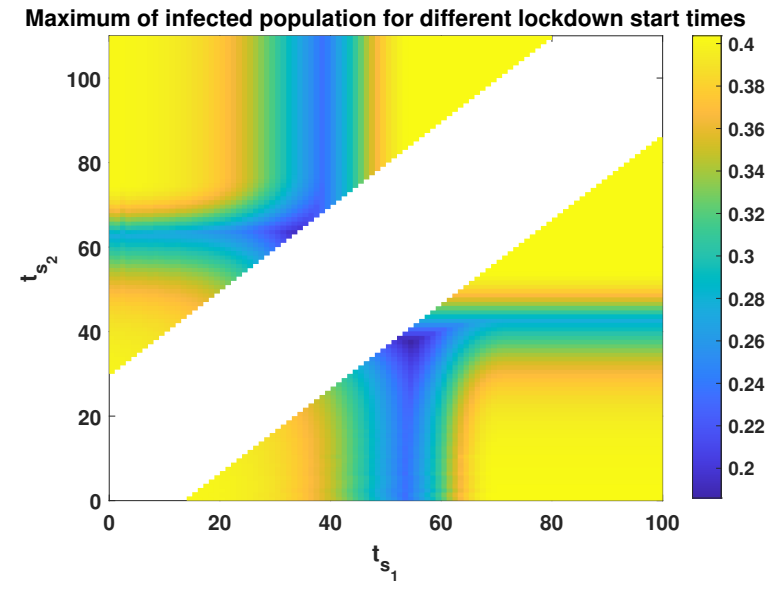

(a) Variation in $I_{\max }$ as a function of $t_{s_{1}}$ and $t_{s_{2}}$

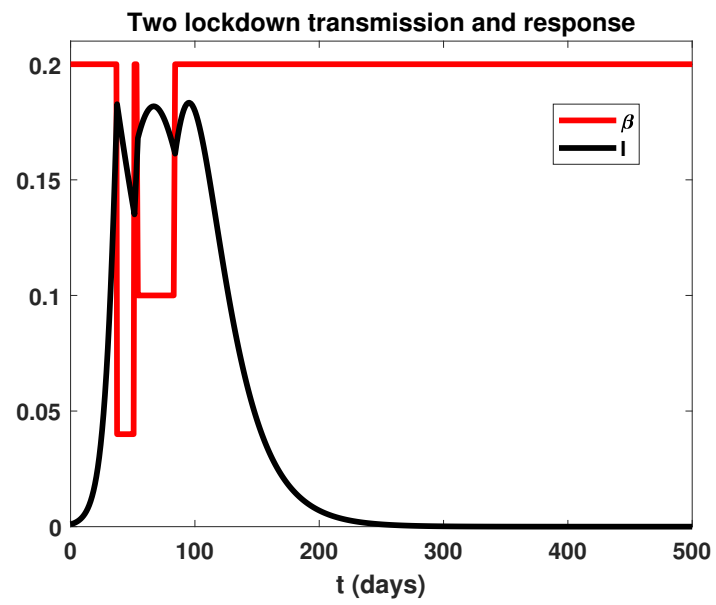

(b) Optimal lockdown schedule and infection response

Figure 4: Numerical investigation of optimal lockdown schedule of the form (17) with $n=2$. Parameters utilized are $\beta=0.2, p_{1}=0.5, p_{2}=0.2, T_{1}=30, T_{2}=14, \nu=0.05, I_{0}=10^{-4}$, and $S_{0}=1-I_{0}$. As in Figure 2, our goal is to minimize the peak of the infected population, which corresponds to bluer shades in Figure 4a. The white region is prohibited, as it would correspond to overalapping lockdowns. The optimal initiation times $t_{s_{1}} \approx 54.1$ days and $t_{s_{2}} \approx 37.4$ days is provided in Figure $4 \mathrm{~b}$. Note that the response $I$ exhibits $3=n+1$ relative maxima (peaks) of equal size. 


\section{Proofs of results}

In this section, we provide proofs of all results stated in the above manuscript, including our main result Theorem 1 . We also present an intermediate proposition (Proposition blah) which allows us to compute the sensitivities of the susceptible population (and also the infected population) with respect to the initiation time $t_{s}$; this is the main tool used to prove Theorem 1.

Proof of Proposition 1. The following calculation is tedious, but elementary. It is well known that the quantity

$$
H(S, I):=I+S-\frac{1}{\mathcal{R}_{0}(\beta)} \log (S)
$$

is conserved along solutions curves with constant $\beta$ and $\nu$. Since transmission rates of the form (4) are piecewise constant, we thus need to consider two two transmission rates: $\beta$ and $p \beta$. The second local maximum of $I$ must occur after the lockdown is released, since $\dot{I} \leq 0$ on $\left(t_{s}, t_{s}+T\right)$. We use repeated use of the conserved quantity $H(S, I)$ on intervals where $\beta(t)$ is constant. Since $I$ is guaranteed to increase after the lockdown is released (assumption (9)), $t_{s}+T$ is a local minimum of $I$, and hence the local maximum must occur on the open interval $\left(t_{s}+T, \infty\right)$. On this interval, $\beta(t) \equiv \beta$ (i.e. $\mathcal{R}_{0}$ is given by $\mathcal{R}_{0}(\beta)$ ), we have the conservation law

$$
H(S(t), I(t))=H\left(S\left(t_{s}+T\right), I\left(t_{s}+T\right)\right)
$$

for $t \geq t_{s}+T$. Thus, we can solve for $I$ as a function of $S$ and the initial conditions $\left(S\left(t_{s}+T\right), I\left(t_{s}+T\right)\right)$ along trajectories:

$$
I=-S+\frac{1}{\mathcal{R}_{0}(\beta)} \log S+I\left(t_{s}+T\right)+S\left(t_{s}+T\right)-\frac{1}{\mathcal{R}_{0}(\beta)} \log S\left(t_{s}+T\right)
$$

A relative maximum of $I$ with respect to $t$ thus corresponds to a relative maximum of $I$ with respect to $S$, which must occur at a susceptible population $S_{*}$ such that $(d I / d S)\left(S_{*}\right)=0$. Utilizing (19) above then implies that $S_{*}$ is given by

$$
S_{*}=\frac{1}{\mathcal{R}_{0}(\beta)} .
$$

Note that $S_{*}$ corresponds to the herd immunity population for the SIR model, and (9) ensures that $S_{*}=S\left(t_{*}\right)$ satisfies

$$
S\left(t_{*}\right)<S\left(t_{s}+T\right)
$$

i.e. that

$$
t_{*}>t_{s}+T
$$

Substituting (20) into (19) yields an expression for the second peak $I_{p}$ of $I$ :

$$
I_{p}=-\frac{1}{\mathcal{R}_{0}(\beta)}\left(1+\log \left(\mathcal{R}_{0}(\beta)\right)\right)+I\left(t_{s}+T\right)+S\left(t_{s}+T\right)-\frac{1}{\mathcal{R}_{0}(\beta)} \log \left(S\left(t_{s}+T\right)\right) .
$$

We now iterate the above procedure on $\left[t_{s}, t_{s}+T\right]$ to simplify the above expression for $I_{p}$ (by simplify, we mean remove as many dependencies on time $t_{s}+T$ as possible). On $\left[t_{s}, t_{s}+T\right], \beta(t) \equiv p \beta$, and the conservation law implies that

$$
H\left(S\left(t_{s}+T\right), I\left(t_{s}+T\right)\right)=H\left(S\left(t_{s}\right), I\left(t_{s}\right)\right) .
$$

Thus, we can solve for $I\left(t_{s}+T\right)+S\left(t_{s}+T\right)$ as

$$
I\left(t_{s}+T\right)+S\left(t_{s}+T\right)=\frac{1}{\mathcal{R}_{0}(p \beta)} \log \left(S\left(t_{s}+T\right)\right)+I\left(t_{s}\right)+S\left(t_{s}\right)-\frac{1}{\mathcal{R}_{0}(p \beta)} \log \left(S\left(t_{s}\right)\right)
$$


Substituting this into (21) yields

$$
\begin{aligned}
I_{p} & =-\frac{1}{\mathcal{R}_{0}(\beta)}\left(1+\log \left(\mathcal{R}_{0}(\beta)\right)\right)+\frac{1}{\mathcal{R}_{0}(p \beta)} \log \left(S\left(t_{s}+T\right)\right) \\
& +I\left(t_{s}\right)+S\left(t_{s}\right)-\frac{1}{\mathcal{R}_{0}(p \beta)} \log \left(S\left(t_{s}\right)\right)-\frac{1}{\mathcal{R}_{0}(\beta)} \log \left(S\left(t_{s}+T\right)\right)
\end{aligned}
$$

Lastly on $\left[0, t_{s}\right]$, where $\beta(t) \equiv \beta$, we have that

$$
H\left(S\left(t_{s}\right), I\left(t_{s}\right)\right)=H\left(S_{0}, I_{0}\right)
$$

which allows us to solve for $I\left(t_{s}\right)+S\left(t_{s}\right)$ as

$$
I\left(t_{s}\right)+S\left(t_{s}\right)=\frac{1}{\mathcal{R}_{0}(\beta)} \log \left(S\left(t_{s}\right)\right)+I_{0}+S_{0}-\frac{1}{\mathcal{R}_{0}(\beta)} \log \left(S_{0}\right),
$$

which yields in (22) the expression

$$
\begin{aligned}
I_{p} & =-\frac{1}{\mathcal{R}_{0}(\beta)}\left(1+\log \left(\mathcal{R}_{0}(\beta)\right)\right)+\frac{1}{\mathcal{R}_{0}(p \beta)} \log \left(S\left(t_{s}+T\right)\right) \\
& +\frac{1}{\mathcal{R}_{0}(\beta)} \log \left(S\left(t_{s}\right)\right)+I_{0}+S_{0}-\frac{1}{\mathcal{R}_{0}(\beta)} \log \left(S_{0}\right) \\
& -\frac{1}{\mathcal{R}_{0}(p \beta)} \log \left(S\left(t_{s}\right)\right)-\frac{1}{\mathcal{R}_{0}(\beta)} \log \left(S\left(t_{s}+T\right)\right) \\
& =\left(\frac{1}{\mathcal{R}_{0}(p \beta)}-\frac{1}{\mathcal{R}_{0}(\beta)}\right) \log \left(\frac{S\left(t_{s}+T\right)}{S\left(t_{s}\right)}\right) \\
& +I_{0}+S_{0}-\frac{1}{\mathcal{R}_{0}(\beta)}\left(1+\log \left(\mathcal{R}_{0}(\beta) S_{0}\right)\right) .
\end{aligned}
$$

We can further simplify (24) by noting that

$$
\begin{aligned}
\frac{1}{\mathcal{R}_{0}(p \beta)}-\frac{1}{\mathcal{R}_{0}(\beta)} & =\frac{1}{p \mathcal{R}_{0}(\beta)}-\frac{1}{\mathcal{R}_{0}(\beta)} \\
& =\frac{1-p}{p} \cdot \frac{1}{\mathcal{R}_{0}(\beta)},
\end{aligned}
$$

and defining the constant $V_{0}$ as in (12), we have that the second relative maximum of $I, I_{p}=I_{p}\left(t_{s}\right)$, is given by

$$
I_{p}\left(t_{s}\right)=V_{0}-\frac{1-p}{p} \cdot \frac{1}{\mathcal{R}_{0}(\beta)} \log \left(\frac{S\left(t_{s}\right)}{S\left(t_{s}+T\right)}\right)+V_{0}
$$

as claimed.

We note that $V_{0}$ is the maximum value of $I$ if a lockdown is never implemented $(\beta(t) \equiv \beta$ on $[0, \infty)$ ), or if the lockdown is "too late" to reduce the peak of the infected population. Equivalently, a peak of $V_{0}$ corresponds to a locktown initiation $t_{s}$ such that

$$
S\left(t_{s}\right) \leq 1 / \mathcal{R}_{0}(\beta)
$$

compare this to (10). In such cases, $I$ has only one (global) maximum $V_{0}$, and is a subset of Case 1 in Section 3.1 (the other disjoint subset is when the lockdown length $T$ is long enough so that the global maximum of $I$ occurs at $t_{s}$, and no other local maxima exist).

Before stating and proving the next proposition, we note that the conserved quantity (18) allows us to transform the twodimensional system (1) into a one-dimensional (nonlinear) ODE if $\beta(t)$ is constant. Specifically, it allows us to solve for $I(t)$ as a function of $S(t)$. For example, on $\left[0, t_{s}\right]$ where $\beta(t) \equiv \beta$, we have that

$$
H(S(t), I(t))=H\left(S_{0}, I_{0}\right),
$$


or equivalently,

$$
I(t)=-S(t)+\frac{1}{\mathcal{R}_{0}(\beta)} \log S(t)+I_{0}+S_{0}-\frac{1}{\mathcal{R}_{0}(\beta)} \log S_{0}
$$

Thus, the dynamics on $\left[0, t_{s}\right]$ can be understood by analyzing the one-dimensional ODE

$$
\begin{aligned}
\dot{S}(t) & =-\beta S(t) I(t) \\
& =-\beta S(t)\left(-S(t)+\frac{1}{\mathcal{R}_{0}(\beta)} \log S(t)+I_{0}+S_{0}-\frac{1}{\mathcal{R}_{0}(\beta)} \log S_{0}\right),
\end{aligned}
$$

with the infected population given by (26). Similarly, on $\left[t_{s}, t_{s}+T\right]$,

$$
\begin{aligned}
\dot{S}(t) & =-p \beta S(t) I(t) \\
& =-p \beta S(t)\left(I_{0}+S_{0}-\frac{1}{\mathcal{R}_{0}(\beta)} \log S_{0}-\frac{1}{\mathcal{R}_{0}(\beta)} \frac{1-p}{p} \log S\left(t_{s}\right)-S(t)+\frac{1}{p \mathcal{R}_{0}(\beta)} \log S(t)\right),
\end{aligned}
$$

where we have used (23) to replace $I\left(t_{s}\right)+S\left(t_{s}\right)$.

Proposition 2. For any fixed $t>0, S=S\left(t ; t_{s}\right)$ is differentiable with respect to the lockdown initiation time $t_{s}$; call this derivative the sensitivity of $S$ with respect to $t_{s}$ at time $t$. Furthermore, we have the following formulas for the sensitivities of $S$ at times $t_{s}$ and $t_{s}+T$ :

$$
\begin{aligned}
\frac{\partial S}{\partial t_{s}}\left(t_{s} ; t_{s}\right) & =-\beta S\left(t_{s}\right) I\left(t_{s}\right) \\
\frac{\partial S}{\partial t_{s}}\left(t_{s}+T ; t_{s}\right) & =-\beta S\left(t_{s}+T\right) I\left(t_{s}+T\right)\left(1+\nu(1-p) I\left(t_{s}\right) \int_{t_{s}}^{t_{s}+T} \frac{\mathrm{d} t}{I(t)}\right) .
\end{aligned}
$$

Proof of Proposition 2. Differentiability is clear. In the following, we suppress the dependence of $S$ on the parameter $t_{s}$ for notational ease. To see (30), we utilize (27), which we write in the form

$$
\frac{d S}{d t}(t)=-\beta S(t) h_{1}(S(t))
$$

with $h_{1}(S):=-S+\frac{1}{\mathcal{R}_{0}(\beta)} \log S+I_{0}+S_{0}-\frac{1}{\mathcal{R}_{0}(\beta)} \log S_{0}$. Note by the argument preceding the proposition we have that $h_{1}(S(t))=I(S(t))$ (see (27)). Since the above is autonomous and one-dimensional, it is separable, and hence we have the relation

$$
\int_{S_{0}}^{S\left(t_{s}\right)} \frac{\mathrm{d} S}{S h_{1}(S)}=-\beta t_{s} .
$$

Differentiating with respect to $t_{s}$ yields the equation

$$
\frac{1}{S\left(t_{s}\right) h_{1}\left(S\left(t_{s}\right)\right)} \frac{\partial S}{\partial t_{s}}\left(t_{s}\right)=-\beta
$$

Thus,

$$
\frac{\partial S}{\partial t_{s}}\left(t_{s}\right)=-\beta S\left(t_{s}\right) h_{1}\left(S\left(t_{s}\right)\right)
$$

As mentioned previously, $h_{1}\left(S\left(t_{s}\right)\right)=I\left(t_{s}\right)$, which yields (30).

We perform the same calculation to obtain (31). That is, write

$$
\frac{d S}{d t}(t)=-p \beta S(t) h_{2}\left(S(t), t_{s}\right)
$$


on $\left[t_{s}, t_{s}+T\right]$, with

$$
h_{2}\left(S, t_{s}\right)=I_{0}+S_{0}-\frac{1}{\mathcal{R}_{0}(\beta)} \log S_{0}-\frac{1}{\mathcal{R}_{0}(\beta)} \frac{1-p}{p} \log S\left(t_{s}\right)-S+\frac{1}{p \mathcal{R}_{0}(\beta)} \log S .
$$

Note that in this case, $h_{2}$ has an additional $t_{s}$ dependence via the $S\left(t_{s}\right)$ term. Separating and integrating (32) yields the relation

$$
\int_{S\left(t_{s}\right)}^{S\left(t_{s}+T\right)} \frac{\mathrm{d} S}{S h_{2}\left(S, t_{s}\right)}=-p \beta T
$$

Differentiating the above (using the Leibniz rule, since $t_{s}$ appears in both the bounds and the integrand), we obtain

$$
\begin{aligned}
\frac{1}{S\left(t_{s}+T\right) h_{2}\left(S\left(t_{s}+T\right), t_{s}\right)} & \frac{\partial S}{\partial t_{s}}\left(t_{s}+T\right)-\frac{1}{S\left(t_{s}\right) h_{2}\left(S\left(t_{s}\right), t_{s}\right)} \cdot \frac{\partial S}{\partial t_{s}}\left(t_{s}\right) \\
& +\int_{S\left(t_{s}\right)}^{S\left(t_{s}+T\right)} \frac{1}{S} \frac{\partial}{\partial t_{s}}\left(\frac{1}{h_{2}\left(S, t_{s}\right)}\right) \mathrm{d} S=0 .
\end{aligned}
$$

The integral term can simplified as follows:

$$
\begin{aligned}
\frac{\partial}{\partial t_{s}}\left(\frac{1}{h_{2}\left(S, t_{s}\right)}\right) & =-\frac{1}{\left(h_{2}\left(S, t_{s}\right)\right)^{2}} \frac{\partial h_{2}}{\partial t_{s}}\left(S, t_{s}\right) \\
& =\frac{1}{\left(h_{2}\left(S, t_{s}\right)\right)^{2}} \cdot \frac{1}{\mathcal{R}_{0}(\beta)} \cdot \frac{1-p}{p} \cdot \frac{1}{S\left(t_{s}\right)} \cdot \frac{\partial S}{\partial t_{s}}\left(t_{s}\right)
\end{aligned}
$$

Hence

$$
\int_{S\left(t_{s}\right)}^{S\left(t_{s}+T\right)} \frac{1}{S} \frac{\partial}{\partial t_{s}}\left(\frac{1}{h_{2}\left(S, t_{s}\right)}\right) \mathrm{d} S=\frac{1}{\mathcal{R}_{0}(\beta)} \cdot \frac{1-p}{p} \cdot \frac{1}{S\left(t_{s}\right)} \cdot \frac{\partial S}{\partial t_{s}}\left(t_{s}\right) \int_{S\left(t_{s}\right)}^{S\left(t_{s}+T\right)} \frac{\mathrm{d} S}{S\left(h_{2}\left(S, t_{s}\right)\right)^{2}}
$$

Recalling that $h_{2}\left(S, t_{s}\right)=I$ on $\left[t_{s}, t_{s}+T\right]$ and changing variables on the integral $(S=S(t)$, as in the first line of (13)), we obtain

$$
\int_{S\left(t_{s}\right)}^{S\left(t_{s}+T\right)} \frac{\mathrm{d} S}{S\left(h_{2}\left(S, t_{s}\right)\right)^{2}}=-p \beta \int_{t_{s}}^{t_{s}+T} \frac{\mathrm{d} t}{I(t)} .
$$

Thus the third term on the left-hand side of (33) becomes

$$
\begin{aligned}
\int_{S\left(t_{s}\right)}^{S\left(t_{s}+T\right)} \frac{1}{S} \frac{\partial}{\partial t_{s}}\left(\frac{1}{h_{2}\left(S, t_{s}\right)}\right) \mathrm{d} S & =(-p \beta) \frac{1}{\mathcal{R}_{0}(\beta)} \cdot \frac{1-p}{p} \cdot \frac{1}{S\left(t_{s}\right)} \cdot \frac{\partial S}{\partial t_{s}}\left(t_{s}\right) \int_{t_{s}}^{t_{s}+T} \frac{\mathrm{d} t}{I(t)} \\
& =-\nu(1-p) \frac{1}{S\left(t_{s}\right)} \cdot \frac{\partial S}{\partial t_{s}}\left(t_{s}\right) \int_{t_{s}}^{t_{s}+T} \frac{\mathrm{d} t}{I(t)} \\
& =\beta \nu(1-p) I\left(t_{s}\right) \int_{t_{s}}^{t_{s}+T} \frac{\mathrm{d} t}{I(t)},
\end{aligned}
$$

where in the final equality we used (30). We can now solve (33) for $\left(\partial S / \partial t_{s}\right)\left(t_{s}+T\right)$, again using (30) and the fact that $h_{2}\left(S\left(t_{s}+T, t_{s}\right)=I\left(t_{s}+T\right), h_{2}\left(S\left(t_{s}\right), t_{s}\right)=I\left(t_{s}\right)\right.$ to obtain

$$
\frac{\partial S}{\partial t_{s}}\left(t_{s}+T\right)=-\beta S\left(t_{s}+T\right) I\left(t_{s}+T\right)-S\left(t_{s}+T\right) I\left(t_{s}+T\right) \beta \nu(1-p) I\left(t_{s}\right) \int_{t_{s}}^{t_{s}+T} \frac{\mathrm{d} t}{I(t)},
$$

which is precisely (31).

Proof of Theorem 1. We have already seen that $I$ must have two relative maxima for $I_{\max }$ to be minimized, and we have also seen that these maxima are given by $I\left(t_{s}\right)$ and $I_{p}\left(t_{s}\right)$. Thus, all that remains to show is that the minimizing $t_{s}$ of (5) must occur when $I\left(t_{s}\right)=I_{p}\left(t_{s}\right)$. Note that it will be sufficient to show that $I\left(t_{s}\right)$ increases as a function of $t_{s}$ 
medRxiv preprint doi: https://doi.org/10.1101/2021.06.26.21259589; this version posted June 30, 2021. The copyright holder for this preprint (which was not certified by peer review) is the author/funder, who has granted medRxiv a license to display the preprint in perpetuity.

All rights reserved. No reuse allowed without permission.

(again, assuming (8)), and that $I_{p}\left(t_{s}\right)$ decreases as a function of $t_{s}$, and that they have a (thus necessarily unique) point of intersection. Note that the latter is clear, assuming $I_{0}$ is small, since $I\left(t_{s}\right) \rightarrow I_{0}$ and $I_{p}\left(t_{s}\right) \rightarrow V_{0}$ as $t_{s} \rightarrow 0$. Hence all that remains is to show the monotonicity properties of $I\left(t_{s}\right)$ and $I_{p}\left(t_{s}\right)$.

Note that is intuitively clear that $I\left(t_{s}\right)$, the value of the first relative maxima of $I$, increases as a function of $t_{s}$, since $(d I / d t)^{-}\left(t_{s}\right)>0$ by (10), so increasing $t_{s}$ increases $I\left(t_{s}\right)$. More rigorously, via the conserved quantity $H$ (see equation (18)), we have that

$$
\begin{aligned}
H\left(S\left(t_{s}\right), I\left(t_{s}\right)\right) & =H\left(S_{0}, I_{0}\right) \\
I\left(t_{s}\right)+S\left(t_{s}\right)-\frac{1}{\mathcal{R}_{0}(\beta)} \log \left(S\left(t_{s}\right)\right) & =I_{0}+S_{0}-\frac{1}{\mathcal{R}_{0}(\beta)} \log \left(S_{0}\right) \\
I\left(t_{s}\right) & =I_{0}+S_{0}-\frac{1}{\mathcal{R}_{0}(\beta)} \log \left(S_{0}\right)-S\left(t_{s}\right)+\frac{1}{\mathcal{R}_{0}(\beta)} \log \left(S\left(t_{s}\right)\right)
\end{aligned}
$$

Taking a derivative with respect to $t_{s}$ of the latter equality yields

$$
\frac{\partial I}{\partial t_{s}}\left(t_{s}\right)=\left(\frac{1}{\mathcal{R}_{0}(\beta) S\left(t_{s}\right)}-1\right) \frac{\partial S}{\partial t_{s}}\left(t_{s}\right)
$$

Since $\mathcal{R}_{0}(\beta) S\left(t_{s}\right)>1$ (this is again (10), which is a consequence of $I$ possessing a local maximum at $t_{s}$ ), it is sufficient to show that $S\left(t_{s}\right)$ decreases as a function of $t_{s}$ at $t=t_{s}$. But this is clear from (30) of Proposition 2.

To prove that $I_{p}\left(t_{s}\right)$ decreases as a function of $t_{s}$, note that by (11), it is sufficient to prove that $S\left(t_{s}\right) / S\left(t_{s}+T\right)$ is increasing as a function of $t_{s}$, or equivalently that $S\left(t_{s}+T\right) / S\left(t_{s}\right)$ is decreasing as a function of $t_{s}$. Since $S$ is differentiable with respect to $t_{s}$ for all fixed $t$, it is sufficient to analyze the sign of the derivative of $S\left(t_{s}+T\right) / S\left(t_{s}\right)$. By elementary calculus, we have that

$$
\frac{\partial}{\partial t_{s}}\left(\frac{S\left(t_{s}+T\right)}{S\left(t_{s}\right)}\right)=\frac{S\left(t_{s}\right) \frac{\partial S}{\partial t_{s}}\left(t_{s}+T\right)-S\left(t_{s}+T\right) \frac{\partial S}{\partial t_{s}}\left(t_{s}\right)}{\left(S\left(t_{s}\right)\right)^{2}}
$$

We can now use formulas (30) and (31) in Proposition 2 to compute the numerator:

$$
\begin{aligned}
S\left(t_{s}\right) \frac{\partial S}{\partial t_{s}}\left(t_{s}+T\right)- & S\left(t_{s}+T\right) \frac{\partial S}{\partial t_{s}}\left(t_{s}\right)=-\beta S\left(t_{s}\right) S\left(t_{s}+T\right) I\left(t_{s}+T\right)\left(1+\nu(1-p) I\left(t_{s}\right) \int_{t_{s}}^{t_{s}+T} \frac{\mathrm{d} t}{I(t)}\right) \\
& +\beta S\left(t_{s}+T\right) S\left(t_{s}\right) I\left(t_{s}\right) \\
& =\beta S\left(t_{s}\right) S\left(t_{s}+T\right) I\left(t_{s}\right) I\left(t_{s}+T\right)\left(\frac{1}{I\left(t_{s}+T\right)}-\frac{1}{I\left(t_{s}\right)}-\nu(1-p) \int_{t_{s}}^{t_{s}+T} \frac{\mathrm{d} t}{I(t)}\right)
\end{aligned}
$$

To complete the proof, we thus need to show that the parenthetical term on the right-hand side of the above is negative. Equivalently, we need to show that

$$
\int_{t_{s}}^{t_{s}+T}\left(\frac{\nu(1-p)}{I(t)}-\frac{d}{d t}\left(\frac{1}{I(t)}\right)\right) \mathrm{d} t>0 .
$$

On $\left(t_{s}, t_{s}+T\right)$, we have that (since $\dot{I}=p \beta S I-\nu I$ )

$$
\begin{aligned}
\frac{\nu(1-p)}{I(t)}-\frac{d}{d t}\left(\frac{1}{I(t)}\right) & =\frac{\nu(1-p)}{I(t)}+\frac{\dot{I}(t)}{I(t)^{2}} \\
& =\frac{1}{I(t)}\left(\nu(1-p)+\frac{p \beta S(t) I(t)-\nu I(t)}{I(t)}\right) \\
& =\frac{p \nu}{I(t)}\left(\mathcal{R}_{0}(\beta) S(t)-1\right) .
\end{aligned}
$$

As $S$ is non-increasing, we have that $S(t) \geq S\left(t_{s}+T\right)$ for all $t \in\left(t_{s}, t_{s}+T\right)$. This together with assumption (9) imply that

$$
\mathcal{R}_{0}(\beta) S(t)>1
$$

for all $t \in\left(t_{s}, t_{s}+T\right)$. Hence the integrand in (36) is positive, which is sufficient to complete the proof. 
medRxiv preprint doi: https://doi.org/10.1101/2021.06.26.21259589; this version posted June 30, 2021. The copyright holder for this preprint (which was not certified by peer review) is the author/funder, who has granted medRxiv a license to display the preprint in perpetuity.

All rights reserved. No reuse allowed without permission.

\section{References}

[1] Seth Flaxman, Swapnil Mishra, Axel Gandy, H Juliette T Unwin, Thomas A Mellan, Helen Coupland, Charles Whittaker, Harrison Zhu, Tresnia Berah, Jeffrey W Eaton, et al. Estimating the effects of non-pharmaceutical interventions on covid-19 in europe. Nature, 584(7820):257-261, 2020.

[2] Linda Thunström, Stephen C Newbold, David Finnoff, Madison Ashworth, and Jason F Shogren. The benefits and costs of using social distancing to flatten the curve for covid-19. Journal of Benefit-Cost Analysis, 11(2):179-195, 2020 .

[3] Olivier Coibion, Yuriy Gorodnichenko, and Michael Weber. The cost of the covid-19 crisis: Lockdowns, macroeconomic expectations, and consumer spending. Technical report, National Bureau of Economic Research, 2020.

[4] Valeria Saladino, Davide Algeri, and Vincenzo Auriemma. The psychological and social impact of covid-19: new perspectives of well-being. Frontiers in psychology, 11:2550, 2020.

[5] Guglielmo Briscese, Nicola Lacetera, Mario Macis, and Mirco Tonin. Compliance with covid-19 social-distancing measures in italy: the role of expectations and duration. Technical report, National Bureau of Economic Research, 2020.

[6] Chris Reinders Folmer, Megan Brownlee, Adam Fine, Malouke Esra Kuiper, Elke Olthuis, Emmeke Barbara Kooistra, Anne Leonore de Bruijn, and Benjamin van Rooij. Social distancing in america: Understanding long-term adherence to covid-19 mitigation recommendations. Amsterdam Law School Research Paper, (2020-62), 2020.

[7] Eduardo D Sontag. An explicit formula for minimizing the infected peak in an sir epidemic model when using a fixed number of complete lockdowns. medRxiv, 2021.

[8] Dylan H Morris, Fernando W Rossine, Joshua B Plotkin, and Simon A Levin. Optimal, near-optimal, and robust epidemic control. Communications Physics, 4(1):1-8, 2021.

[9] Francesco Di Lauro, István Z Kiss, and Joel C Miller. Optimal timing of one-shot interventions for epidemic control. PLoS Computational Biology, 17(3):e1008763, 2021.

[10] Alex LK Morgan, Mark EJ Woolhouse, Graham F Medley, and Bram AD van Bunnik. Optimizing time-limited non-pharmaceutical interventions for covid-19 outbreak control. Philosophical Transactions of the Royal Society B, 376(1829):20200282, 2021.

[11] Mahdiar Sadeghi, James M Greene, and Eduardo D Sontag. Universal features of epidemic models under social distancing guidelines. Annual reviews in control, 2021.

[12] Marco Tulio Angulo, Fernando Castaños, Rodrigo Moreno-Morton, Jorge X Velasco-Hernández, and Jaime A Moreno. A simple criterion to design optimal non-pharmaceutical interventions for mitigating epidemic outbreaks. Journal of the Royal Society Interface, 18(178):20200803, 2021.

[13] Pierre-Alexandre Bliman and Michel Duprez. How best can finite-time social distancing reduce epidemic final size? Journal of theoretical biology, 511:110557, 2021.

[14] Pierre-Alexandre Bliman, Michel Duprez, Yannick Privat, and Nicolas Vauchelet. Optimal immunity control and final size minimization by social distancing for the sir epidemic model. Journal of Optimization Theory and Applications, 189(2):408-436, 2021.

[15] AH González, AL Anderson, A Ferramosca, and EA Hernandez-Vargas. Dynamic characterization of control sirtype systems and optimal single-interval control. arXiv preprint arXiv:2103.11179, 2021.

[16] William Ogilvy Kermack and Anderson G McKendrick. A contribution to the mathematical theory of epidemics. Proceedings of the royal society of london. Series A, Containing papers of a mathematical and physical character, 115(772):700-721, 1927. 
medRxiv preprint doi: https://doi.org/10.1101/2021.06.26.21259589; this version posted June 30, 2021. The copyright holder for this preprint (which was not certified by peer review) is the author/funder, who has granted medRxiv a license to display the preprint in perpetuity. All rights reserved. No reuse allowed without permission.

[17] Jan M Brauner, Sören Mindermann, Mrinank Sharma, David Johnston, John Salvatier, Tomáš Gavenčiak, Anna B Stephenson, Gavin Leech, George Altman, Vladimir Mikulik, et al. Inferring the effectiveness of government interventions against covid-19. Science, 371(6531), 2021.

[18] Notes on mathematical systems biology.

https://drive.google.com/drive/folders/ 11 IRqaCPeXMVZGoY-4 4bBsvtnsHt lRfIO, 2021. 